\title{
An Assessment of Computational Methods for Obtaining Structural Information of Moderately Flexible Biomolecules from Ion Mobility Spectrometry
}

\author{
Natalia L. Zakharova, Christina L. Crawford, Brian C. Hauck, Jacob K. Quinton, \\ William F. Seims, Herbert H. Hill Jr, Aurora E. Clark \\ Department of Chemistry, Washington State University, Pullman, WA 99164, USA
}

\begin{abstract}
When utilized in conjunction with modeling, the collision cross section $(\Omega)$ from ion mobility spectrometry can be used to deduce the gas phase structures of analyte ions. Gas phase conformations are determined computationally, and their $\Omega$ calculated using an approximate method, the results of which are compared with experimental data. Though prior work has focused upon rigid small molecules or large biomolecules, correlation of computational and experimental $\Omega$ has not been thoroughly examined for analytes with intermediate conformational flexibility, which constitute a large fraction of the molecules studied in the field. Here, the computational paradigm for calculating $\Omega$ has been tested for the tripeptides WGY, YGW, and $Y W G(Y=$ tyrosine, $W=$ tryptophan, $G=$ glycine). Experimental data indicate that $\Omega_{\exp }(Y W G)>\Omega_{\exp }(W G Y) \approx \Omega_{\exp }(Y G W)$. The energy distributions of conformations obtained from tiers of simulated annealing molecular dynamics (SAMD) were analyzed using a wide array of density functionals. These quantum mechanical energy distributions do not agree with the MD data, which leads to structural differences between the SAMD and DFT conformations. The latter structures are obtained by reoptimization of the SAMD geometries, and are the only suite of structures that reproduce the experimental trend in analyte separability. In the absence of fitting Lennard Jones potentials that reproduce experimental results for the Trajectory Method, the Exact Hard Sphere Scattering method produced numerical values that are in best agreement with the experimental cross sections obtained in He drift gas.
\end{abstract}

Key words: Ion mobility-mass spectrometry, Collision cross section, Density functional theory, MOBCAL, Simulated annealing molecular dynamics

\section{Introduction}

on mobility spectrometry (IMS) is an analytical method that is unique in its ability to separate structural, geometric, and

Electronic supplementary material The online version of this article (doi:10.1007/s13361-012-0339-5) contains supplementary material, which is available to authorized users.

Correspondence to: Aurora E. Clark; e-mail: auclark@wsu.edu enantiomeric isomers in the gas phase by measuring the ion's mobility in a neutral drift gas in the presence of a uniform electric field [1-3]. In turn, the mobility can be used to determine the collision cross section $\left(\Omega_{\text {exp }}\right)$, a measure of an ion's conformation in the gas phase [4]. The combination of mobility and mass separation using ion mobility-mass spectrometry (IM-MS) [5] has made IMS an important tool in emerging scientific fields that include proteomics [2, 6, 7], metabolomics [8], and glycomics [9]. Gas phase separation occurs in IMS by repeated collisions of the gas phase ion with 
neutral drift gas molecules. Ions of identical composition but different conformations may be separated because the number of collisions is dependent on the analyte's collision cross section $\left(\Omega_{\text {exp }}\right)$. If utilized in conjunction with computational modeling, comparisons between theoretical and experimental data can be used to deduce the gas phase structures of analyte ions.

The computational component consists of two parts; first determining gas phase structures that are likely present at the experimental temperature, followed by the application of an approximate method to calculate the theoretical $\Omega$ (e.g., the projection approximation (PA), the exact hard-sphere scattering approximation (EHSS), or the trajectory method (TM)). In typical applications, hundreds of structures are generated and a graph of energy versus theoretical $\Omega$ reveals low energy structures with $\Omega$ values that agree well with experiment. Yet in principle, the most stable, thermally populated conformations should de facto have cross sections relevant to the experimental data. To determine accurate gas phase geometries, a number of approaches may be taken. When the analyte has few internal degrees of freedom, or is very rigid, temperature independent methods like density functional theory (DFT) may be used [10]. Molecular dynamics (MD) or tiers of simulated annealing molecular dynamics (SAMD) is more appropriate for molecules with conformational flexibility, and can be used to determine an ensemble of thermally populated configurations [11-18]. However, there are a number of computational hurdles associated with calculating the structures present within the IMS drift tube. One area of effort has been dedicated toward accurate sampling of the MD configurations so as to best represent an ensemble that is present under the millisecond time regime of the IMS experiment [19-21]. This is particularly relevant for large biomolecules that may be folding and unfolding during the drift time. For molecules with an intermediate amount of flexibility, many questions remain regarding the accuracy of computationally predicted structures and their relative energetics (which is key to determining the thermally populated conformations). To our knowledge, there have been no systematic studies regarding the dependence of the geometric structures and their relative energetic ranking upon computational methods or simulation protocols for moderately flexible analytes.

The objective of the present work is to critically examine the computational paradigm for calculating $\Omega$ using molecular modeling methods. We are particularly interested in the ability of a typical user to utilize SAMD to a priori identify the relevant 'low-energy' conformations fed into $\Omega$ calculations of moderately flexible analytes, as well as the performance of the different methods for approximating $\Omega$ as a function of the drift gas conditions. Toward this end, three tripeptide isomers, WGY, YGW, and YWG (Y= tyrosine, $\mathrm{W}=$ tryptophan, $\mathrm{G}=$ glycine), have been studied experimentally and computationally. These tripeptides exhibit varying levels of separation as a function of drift gas in the IMS experiments described below, and contain on the order of 10 freely rotating bonds, imparting a modest level of conformational flexibility.

\section{Experimental}

\section{Theory of IMS}

In IMS, a sample is detected by generating thermalized, gas phase ions in an electric field. Mobility separation begins by pulsing a fraction of the sample ions into the IMS drift region via an electronic ion gate. Within conventional, atmospheric pressure IMS, the ions then drift down a uniform electric field $\left(E, \mathrm{Vcm}^{-1}\right)$ at a characteristic velocity $\left(v, \mathrm{~cm} \mathrm{~s}^{-1}\right)$ in the presence of a counter-current drift gas and are separated in time due to their differing ion velocities and detected according to their ionic mobility $\left(K, \mathrm{~cm}^{2} \mathrm{~V}^{-1} \mathrm{~s}^{-1}\right)$ :

$$
K=\frac{v_{d}}{E}=\frac{L^{2}}{V t_{d}}
$$

where $t_{d}$ is the ion's drift time (seconds) as it travels a distance $L(\mathrm{~cm})$ along a drift tube with a potential difference of $V$ (volts). Since IMS systems are built in various configurations and used in diverse operating environments, the more useful reduced mobility $\left(K_{0}, \mathrm{~cm}^{2} \mathrm{~V}^{-1} \mathrm{~s}^{1}\right)$ value corrects $K$ for temperature and pressure differences and is a more useful value to compare data across various systems [22]:

$K_{0}=K\left(\frac{273.15}{T}\right)\left(\frac{P}{760}\right)=\left(\frac{L^{2}}{V t_{d}}\right)\left(\frac{273.15}{T}\right)\left(\frac{P}{760}\right)$.

An ion's mobility is related to its size and charge through an expression first used by Revercomb and Mason [23]:

$$
\frac{\Omega_{\exp }}{z}=\left(\frac{3}{16 N}\right)\left(\frac{2 \pi}{\mu k T}\right)^{\frac{1}{2}}\left(\frac{e}{K}\right)
$$

where $e$ is the charge on an electron $\left(1.602 \times 10^{-19} \mathrm{C}\right), z$ is the number of charges on the ion, $N$ is the number density of the drift gas, $\mu$ is the reduced mass of the ion-neutral drift gas pair, $k$ is Boltzmann's constant, $K$ is the ion's mobility $\left(\mathrm{cm}^{2} \mathrm{~V}^{-1} \mathrm{~s}^{-1}\right)$, and $\Omega$ is the collision cross section of the ion (in $\AA^{2}$ ).

The ability to separate peaks in IMS is calculated using the single-peak definition of the measured resolving power $\left(R_{m}\right)$ :

$$
R_{m}=\frac{t_{d}}{w}
$$

where $t_{d}$ is the drift time of the ion species and $\mathrm{w}$ is the peak width at half-height [24-27]. The resolving power of an IMS is dependent upon the electric field homogeneity, diffusion, ion gate pulse width, Coulombic repulsion, and amplifier response or 'rise' time. The degree of separation between 
two closely spaced mobility peaks can be expressed using a separation factor $(\alpha)[27,28]$. Similar to the separation factor used in chromatography, the IMS separation factor is:

$$
\alpha=\frac{K_{1}}{K_{2}}
$$

where $K_{1}$ is the mobility of the faster drifting compound and $K_{2}$ is the mobility of the slower drifting compound. Separation factors are always greater than or equal to 1 ; a value of 1 indicates that the mobility peaks cannot be separated by the current method's resolving power.

\section{Chemicals and Materials}

HPLC-grade solvents were purchased from Mallinckrodt Baker, Inc. (Philipsburg, NJ, USA). Certified ACS-grade glacial acetic acid was purchased from Fisher Chemical (Fairlawn, NJ, USA). IMS standard compounds 2,4-lutidine and di-tert-butylpyridine (DtBP) were purchased from Sigma-Aldrich Inc. (St. Louis, MO, USA). The isobaric peptides WGY, YGW, and YWG were synthesized at WSU using Fmoc chemistry on an Applied Biosystems (Foster City, CA, USA) 431A peptide synthesizer per the manufacturer's instructions. The Fmoc amino acids were purchased from Novabiochem (Gibbstown, NJ, USA). Five hundred $\mu \mathrm{M}$ solutions of each peptide and a mixture of the three peptides were made up in the ESI spray solution. The three mobility drift gases used in this study, carbon dioxide, nitrogen, and helium, were purchased from A-L Compressed Gases, Inc. (Spokane, WA, USA). All chemicals and gases were used without further purification.

\section{Instrumentation}

The ion mobility-time of flight mass spectrometer (IMTOFMS) with accompanying electrospray ionization (ESI) source has previously been described in detail and demonstrated the ability to reproduce previously reported and accepted mobility values for a variety of analytes [29-31]. Samples were introduced into the IM-TOFMS via an ESI source constructed in-house using polyimide coated fused silica capillary (i.d.: $50 \mu \mathrm{m}$; o.d.: $150 \mu \mathrm{m}$ ) purchased from Polymicro (Phoenix, AZ, USA) and stainless-steel fittings from VICI Valco (Houston, TX, USA). The electrospray solvent was sprayed at a flow rate of $3 \mu \mathrm{L} \min ^{-1}$ and consisted of $5 \%$ glacial acetic acid in equal parts methanol and water. A countercurrent flow of $\mathrm{N}_{2}, \mathrm{CO}_{2}$, or He drift gas was introduced near the rear of the IMS from a compressed gas cylinder (set to $50 \mathrm{psig}$ with attached regulator) according to the methods first used by Beegle et al. [32]. The $\mathrm{N}_{2}$ and $\mathrm{CO}_{2}$ gases were introduced at a flow rate of $1 \mathrm{~L}$ gas $\mathrm{min}^{-1}$ by an Aalborg "P" Series rotameter (Orangeburg, NY, USA) connected by $1 / 4$ in. stainless steel tubing to the gas cylinder using Swagelok fittings (Solon, OH, USA). The He drift gas was introduced into the mobility drift space in the same manner described above but at a flow rate twice as large as the $\mathrm{N}_{2}$ or $\mathrm{CO}_{2}$ and regulated to $80 \mathrm{psi}$. The IMS was operated at $200{ }^{\circ} \mathrm{C}(473.15 \mathrm{~K})$ in a similar manner previously described. Response ions were generated by the ESI source in the $3.0 \mathrm{~cm}$ ion-molecule reaction region and then subsequently pulsed into the $16.5 \mathrm{~cm}$ drift region by a Bradbury-Nielsen ion shutter with a $400 \mu$ s pulse width. The ions traveled under a uniform electric field of $590 \mathrm{~V} \mathrm{~cm}^{-1}$ (429 $\mathrm{V} \mathrm{cm}^{-1}$ with the He drift gas) towards the TOFMS at ambient pressure (approximately 700 Torr); the ions then traversed a $300 \mu \mathrm{m}$ pin hole leak into a pressure interface region (1.6 Torr) and ion focusing region $\left(2.5 \times 10^{-2}\right.$ Torr) of the TOFMS. A series of ion lenses pushed the ions towards the TOF extraction pulser where orthogonal extraction introduced the ions into the $\mathrm{V}$-shaped flight path of the TOFMS $\left(1.9 \times 10^{-6}\right.$ Torr). A Burle (Lancaster, PA, USA) multi-channel plate detector was used for response ion detection. Spectra were generated by software developed at Ionwerks Inc. (Houston, TX, USA) which is run on the ITT Visual Information Solutions IDL Virtual Machine platform (Boulder, CO, USA).

\section{Reproducibility of Data}

Data were generated for each peptide on the IM-TOFMS by collecting multiple ion mobility- mass spectra (IMMS). After each data collection run, the fused silica ESI capillary and sample syringe were cleaned with successive additions of water, dichloromethane, and methanol to prevent carryover. A mass spectrum was collected with a blank sample of the ESI spray solution to confirm the absence of the previous peptide. The above procedure was repeated for each peptide in each of the three drift gases. These procedures were then repeated over multiple days. At the end of each day, IMMS spectra were collected for the mobility standards 2,4-lutidine and di-tert-butyl pyridine (D $t \mathrm{BP})$.

\section{Computational Details}

Initial gas phase geometry optimizations of WGY, YGW, and YWG tripeptides in varying protonation states (Figure 1) were performed using DFT with the B3LYP exchange correlation functional $[33,34]$ and the $6-31(\mathrm{~g}) \mathrm{d}$ basis set [35] using the Gaussian 03 electronic structure program [36]. While the B3LYP functionals do not include dispersion interactions, this method is the most often used within the literature, and we show (vide infra) that the lack of dispersion does not influence the predicted geometries for the specific molecules under consideration. In certain cases, the aug-cc-pVDZ basis [37] was used for geometry optimization to verify the quality of the $6-31 \mathrm{G}^{*}$ structures. Single point aug-cc-pVDZ calculations were also performed so as to compare the dependence of the relative energy ranking of structures as a function of basis set. Normal mode coordinate analysis confirmed all structures to be local minima with no imaginary vibrational frequencies. The 
<smiles>COc1ccc(CCNC=O)cc1</smiles>

WGY<smiles></smiles>

YGW<smiles>[B]c1ccccc1CCNC(=O)NCCc1ccc(OC)cc1</smiles>

YWG

Figure 1. Structures for tripeptides WGY, YGW, and YWG with protonation sites indicated by capital letters. The A-state designates the $\mathrm{N}$-terminus, the $\mathrm{B}$-state designates the tryptophan side chain, and the C-state designates the tyrosine side chain as well as a number of zwitterionic states.

resulting structures were used as starting points for simulated annealing molecular dynamics (SAMD) simulations in the CHARMM program [38] to determine structures populated under the experimental temperature of $473 \mathrm{~K}$.

The SAMD simulation consists of multiple steps wherein the initial structure (the "seed" geometry) is heated and equilibrated at a temperature that is much higher than experiment. This allows the analyte molecule to explore its conformational potential energy surface (PES). Next, the temperature is slowly lowered to the experimental value. Within the distribution of conformations under the new temperature, the statistically dominant conformations are recorded and their energies calculated so as to identify the lowest energy geometry, which is then saved and used as the seed in the next SAMD cycle. In typical applications enough cycles are run so as to generate 100-200 structures, whereupon diagnostic graphs of energy versus $\Omega$ are generated. The low- energy structures whose calculated $\Omega$ values agree with experiment are then chosen for further study and analysis [39-41]. For moderately flexible analytes, like the tripeptides studied here, the number of available conformations is limited, and thus in this work the SAMD cycles were repeated until a self-consistent distribution of structures (typically in the 10s of cycles) was obtained.

While the specific SAMD protocols used within the literature vary, it is important to note that (to our knowledge) no study has directly compared the conformational distributions of two different protocols within the same work. Two SAMD protocols were used so as to investigate the role of equilibration, heating, and cooling times upon the conformational distributions. Each simulation consisted of 20 cycles, beginning with 10,000 steps of minimization. The peptide was then heated from 0 to $1000 \mathrm{~K}$. Following equilibration, the peptide was cooled to the experimental temperature of $473 \mathrm{~K}$ and re-equilibrated. Structures adopted by the peptide at $473 \mathrm{~K}$ were recorded every 100 steps thereafter and the lowest energy geometry was then used as the seed structure in the next simulated annealing cycle. This overall protocol is typical of that in the literature, however less than 50 ps of equilibration at the elevated temperature is generally performed, followed by less than $50 \mathrm{ps}$ of a cooling period and re-equilibration and or re-minimization [39-41]. Though the total length of the simulation is somewhat limited by the size of the analyte and the computational resources, the relatively small size of the tripeptides studied here enables much longer equilibration times than typically used. While it might be assumed that long equilibration times would lead to similar conformational distributions, the data still shows a high level of sensitivity to this aspect of the simulation protocol (vide infra). Simulated annealing protocol \#1 consisted of 5000 ps of initial heating to $1000 \mathrm{~K}$, followed by 3000 ps of equilibration, then 20,000 ps of cooling to $473 \mathrm{~K}$, and 1000 ps of equilibration at $473 \mathrm{~K}$; structures were recorded for a total of 10,000 ps [17]. Simulated annealing protocol \#2 consisted of 500 ps of initial heating to $1000 \mathrm{~K}$, followed by 300 ps of equilibration, then 2,000 ps of cooling to $473 \mathrm{~K}$, and $100 \mathrm{ps}$ of equilibration at $473 \mathrm{~K}$; structures were recorded for a total of 10,000 ps. Both protocols used a 1 fs time step for all stages of simulated annealing. Both 'compact' ( $\mathrm{N}$ - and C-termini close in proximity) and 'elongated' (N- and C-termini far apart) seed geometries were used.

To assess the energetic differences between the SAMD structures, single point DFT calculations were performed on the five lowest energy structures from protocol \#1 for each protonation state of WGY. Calculations using the generalized gradient (GGA) functional PBE [42], BLYP [33, 35], the metaGGA functionals TPSS [43], and VSXC [44], as well as the hybrid functional B3LYP [33], PBE1PBE [45], and the hybrid meta-GGA functional TPSSH $[43,46]$ were employed to gauge which functional most closely agreed with the classical energy distribution. The PBE, TPSS, and TPSSH functionals 
were performed using the NWChem [47] electronic structure program, while all other calculations used Gaussian 03. Finally, the lowest energy SAMD geometries were reoptimized using DFT with B3LYP and the 6-31G* basis set.

The collision cross sections were calculated at $473 \mathrm{~K}$ using the Projection Approximation, Exact Hard Sphere Scattering, and the Trajectory Method, as implemented in the MOBCAL software package [48, 49]. The Projection Approximation (PA) (eq 6) determines the cross section of a spherical system, $\Omega_{\mathrm{PA}}$, by taking an average over geometric cross sections for plausible collision geometries [11, 50-52]:

$$
\Omega_{P A}=\frac{1}{8 \pi^{2}} \int_{0}^{2 \pi} d \theta \int_{0}^{\pi} d \phi \sin \phi \int_{0}^{2 \pi} d \gamma \pi b_{\min }^{2}
$$

where $\theta, \varphi, \gamma$ are Euler angles that define the collision geometry between the polyatomic ion and the drift gas atom and $b_{\min }$ is the hard-sphere contact distance. The Exact Hard Sphere Scattering (EHSS) approximation (eq 7) determines the cross section of a spherical system, $\Omega_{\mathrm{EHSS}}$, using the angle between incoming and outgoing trajectories [49]:

$\Omega_{E H S S}=\frac{1}{4 \pi^{2}} \int_{0}^{2 \pi} d \theta \int_{0}^{\pi} d \phi \sin \phi \int_{0}^{2 \pi} d \gamma \int_{0}^{\infty} d b 2 b(1-\cos \chi(\theta, \phi, \gamma, b))$

where $\theta, \varphi, \gamma$ are Euler angles used to define the collision geometry for the analyte and the drift gas, $\chi(\theta, \varphi, \gamma, b)$ is the scattering angle, $b$ is the impact parameter, and $\mu$ is the reduced mass. As in the EHSS method, the Trajectory Method (TM) (eq 8) uses the angle between incoming and outgoing trajectories to determine the cross section of a spherical system $\Omega_{\mathrm{TM}}$. However, the TM also incorporates a Boltzman distribution of drift gas velocities at a specified temperature:

$$
\begin{aligned}
\Omega_{T M}= & \frac{1}{8 \pi^{2}} \int_{0}^{2 \pi} d \theta \int_{0}^{\pi} d \phi \sin \phi \int_{0}^{2 \pi} d \gamma \frac{\pi}{8} \\
& \times\left(\frac{\mu}{k_{B} T}\right)^{3} \int_{0}^{\infty} d g e^{\frac{-\mu g^{2}}{2 k_{B} T}} g^{5} \int_{0}^{\infty} d b 2 b \\
& \times(1-\cos \chi(\theta, \phi, \gamma, b))
\end{aligned}
$$

where $\theta, \varphi, \gamma$ are Euler angles that define the collision geometry for the analyte ion and the drift gas atom, $\chi(\theta, \varphi, \gamma$,

Table 1. Lennard-Jones (LJ) Parameters used in MOBCAL [57]

\begin{tabular}{llrrc}
\hline Gas & \multirow{2}{*}{$\begin{array}{l}\text { Polarizability } \\
\left(\times 10^{-30} \mathrm{~m}^{3}\right)\end{array}$} & Mass (au) & \multicolumn{2}{c}{ LJ parameters } \\
\cline { 3 - 5 } & & & $\varepsilon_{\mathrm{o}} / \mathrm{k}(\mathrm{K})$ & $\mathrm{r}_{\mathrm{o}}\left(\times 10^{-10} \mathrm{~m}\right)$ \\
\hline $\mathrm{He}$ & 0.204956 & 4.0026 & 15.55 & 3.043 \\
$\mathrm{CO}_{2}$ & 2.911 & 44.0095 & 201.71 & 4.444 \\
$\mathrm{~N}_{2}$ & 1.7403 & 28.0134 & 91.85 & 3.919 \\
\hline
\end{tabular}

$g, b)$ is the scattering angle, $g$ is the relative velocity of the drift gas, $b$ is the impact parameter defining how far the drift gas atom is positioned from the $\mathrm{x}$-axis, $\mu$ is the reduced mass, $k_{B}$ is Boltzmann's constant, and $T$ is temperature in Kelvin. The collisions between drift gas and analyte are also modulated by an interaction potential between the drift gas molecule and atoms in the analyte ion. In this work, both a polarization term and a 12-6 Lennard-Jones potential is used [5]. In eqs $6-8$, only fully elastic collisions occur between the drift gas and analyte[53].

The TM $\Omega$ values used Lennard-Jones (LJ) parameters for $\mathrm{He}, \mathrm{CO}_{2}$, and $\mathrm{N}_{2}$ drift gases (Table 1). While the LJ parameters in MOBCAL have been optimized for the pairwise interactions of $\mathrm{He}$ with the elements in a biomolecule and are traditionally unaltered within the literature [18], the values for $\mathrm{CO}_{2}$ and $\mathrm{N}_{2}$ were taken from the optimized parameters of $\mathrm{CO}_{2}$ and $\mathrm{N}_{2}$ gas over a wide temperature and pressure range [54]. The interaction between analyte and drift gas is described in MOBCAL by a superposition of $\mathrm{LJ}$ potentials (in addition to the chargeinduced dipole interaction) each describing the interaction of one atom in the analyte with the drift gas. No attempt has been made to tailor the $\mathrm{LJ}$ terms for $\mathrm{CO}_{2}$ and $\mathrm{N}_{2}$ to reproduce cross sections for biomolecules, and as such we do not anticipate these potentials to reproduce the magnitude of the drift gas dependency of $\Omega$. Rather the trend in $\Omega$ as a function of drift gas should be reproduced, as this is largely determined by the polarizability of the drift gas. Further, the relative order of the collision cross sections as a function of the specific geometry of the tripeptide will not be altered by the lack of optimization of the $\mathrm{CO}_{2}$ and $\mathrm{N}_{2} \mathrm{LJ}$ potentials, since the dominant contribution to $\Omega$ is the molecular geometry itself. The number of trajectories for the TM and EHSS calculations was 600,000 and $18,000,000$, respectively, while the number of sample points for the PA calculation was $18,000,000$. In instances where several structures were predicted to be thermally populated under the experimental conditions (with energy differences less than $2.0 \mathrm{kcal} / \mathrm{mol}$ ), the collision cross sections of both the individual structures as well as the average values are presented, as in prior work $[55,56]$. Mulliken atomic charges were used to create the charge distribution of the analyte and were taken from single point B3LYP/6-31G* DFT calculations.

\section{Results and Discussion}

\section{Analysis of Isomeric Peptides by (+)ESI-IM-TOFMS}

The 3-day average $(n=15)$ reduced mobility $\left(K_{0}\right)$ values for each peptide in each drift gas are shown in Table 2. Overall, the $K_{0}$ values for all of the peptides were largest in $\mathrm{He}$ followed by those values obtained in $\mathrm{N}_{2}$ and $\mathrm{CO}_{2}$ drift gases, respectively. The $K_{0}$ values for the peptides followed the trend $\mathrm{YGW}>\mathrm{WGY}>\mathrm{YWG}$. 
Table 2. $K_{0}$ Values $\left(\mathrm{cm}^{2} \mathrm{~V}^{-1} \mathrm{sec}^{-1}\right)$ and Average Measured Resolving Power $(n=4)$ for WGY, YGW, and YWG in $\mathrm{He}, \mathrm{N}_{2}$, and $\mathrm{CO}_{2}$ Drift Gases

\begin{tabular}{llllrr}
\hline Peptide & $K_{0}(\mathrm{He})$ & $R m_{H e}$ & $K_{0}\left(\mathrm{~N}_{2}\right)$ & $R m_{N 2}$ & $K_{0}\left(\mathrm{CO}_{2}\right)$ \\
\hline WGY & $3.15 \pm 0.03$ & $48 \pm 23$ & $0.970 \pm 0.002$ & $84 \pm 26$ & $0.660 \pm 0.002$ \\
YGW & $3.19 \pm 0.04$ & $39 \pm 9$ & $0.990 \pm 0.002$ & $125 \pm 17$ & $0.680 \pm 0.010$ \\
YWG & $3.00 \pm 0.02$ & $40 \pm 7$ & $0.940 \pm 0.003$ & $80 \pm 7$ & $0.630 \pm 0.003$ \\
\hline
\end{tabular}

Table 3 shows the collision cross sections, $\Omega_{\text {exp }}$, found for each peptide in each drift gas; the $\Omega_{\exp }$ were found for a hypothetical zero polarizability drift gas by extrapolation from the $\Omega_{\exp }$ values found in the $\mathrm{He}, \mathrm{N}_{2}$, and $\mathrm{CO}_{2}$ drift gases. This extrapolation is based on prior empirical observations that an inverse relationship exists between the reduced mobility constants and the mass and polarizability of the neutral drift gas [57]. The differing slopes of the extrapolation plots indicate that the ionic collision partner adds its own "polarizing power" to the polarizability of the neutral gas to yield an experimental cross section that is an intertwined property of both interacting species: YGW has a lower polarizing power than WGY or YWG. Difference in polarizing power can actually invert the IMS elution order of ionic species in different drift gases [28]. The zero polarizability $\Omega_{\exp }$ can be thought of as the cross section that would be observed for each peptide colliding with a very small, unpolarizable neutral, and in conjunction with polarizing power can be taken as measure of the peptide ion's size summarizing more data than a single-gas cross section. We anticipate the zero polarizability cross section to have the most similarity to $\Omega_{\mathrm{PA}}$ and $\Omega_{\mathrm{EHSS}}$ as the mass and size of the drift gas, as well as any potential energy interaction between drift gas and analyte is ignored within those methods. Figure 2 shows the ion mobility-mass spectra of YWG, WGY, and YGW $\left(m / z 425,[\mathrm{M}+\mathrm{H}]^{+}\right)$in $\mathrm{He}, \mathrm{N}_{2}$, and $\mathrm{CO}_{2}$ drift gases. The mobility spectra show clear separation between the pairs of peptides. However, the separation factors $(\alpha)$ between the YGW and WGY peptide pair (bottom spectra) is smaller $\left(\alpha_{\mathrm{He}}: 1.01 ; \alpha_{\mathrm{N} 2}: 1.02 ; \alpha_{\mathrm{CO} 2}\right.$ : 1.03) than either the YWG and YGW pair (middle spectra, $\left.\alpha_{\mathrm{He}}: 1.06 ; \alpha_{\mathrm{N} 2}: 1.05 ; \alpha_{\mathrm{CO} 2}: 1.08\right)$ or the YWG and WGY pair (top spectra, $\alpha_{\mathrm{He}}: 1.05 ; \alpha_{\mathrm{N} 2}: 1.03 ; \alpha_{\mathrm{CO} 2}: 1.05$ ). YWG is clearly separated from the other two peptides while YGW and WGY had very similar $K_{0}$ and $\Omega_{\exp }$ values in each of the

Table 3. Collision Cross Section Values, $\Omega_{\exp }$ (in $\AA^{2}$ ), Calculated from $K_{0}$ in Table 2 (The Number of Samples was $n=15$ with Error Reported to $1 \sigma$ )

\begin{tabular}{llllll}
\hline Peptide & $\begin{array}{l}\Omega_{\exp } \text { zero } \\
\text { polarizability }\end{array}$ & $\begin{array}{l}\text { Polarizing } \\
\text { power }\left(\AA^{-1}\right)\end{array}$ & $\Omega_{\exp }(\mathrm{He})$ & $\Omega_{\exp }\left(\mathrm{N}_{2}\right)$ & $\Omega_{\exp }\left(\mathrm{CO}_{2}\right)$ \\
\hline WGY & 118 & 25.7 & $135.0 \pm 1.5$ & $170.0 \pm 0.2$ & $205.0 \pm 1.5$ \\
YGW & 118 & 24.2 & $134.0 \pm 1.6$ & $167.0 \pm 0.4$ & $200.0 \pm 3.1$ \\
YWG & 124 & 25.7 & $143.0 \pm 1.1$ & $177.0 \pm 0.4$ & $213.0 \pm 0.6$ \\
\hline
\end{tabular}

${ }^{\text {a }}$ The $\Omega_{\exp }$ in a hypothetical zero polarizability drift gas was obtained by plotting the $\Omega_{\exp }$ values against the drift gas polarizability for each gas and finding the intercept of the resulting line.

"Polarizing power" is the slope of this plot. drift gases. In a survey of peak widths for WGY, the peak widths were significantly wider than that of a nearby peak in the experimental mobility spectrum for the $\mathrm{He}$ and $\mathrm{CO}_{2}$ drift gases, indicating non-interconverting conformations with nearly identical cross section values. Similar results were observed for YGW in $\mathrm{N}_{2}$ and YWG in both $\mathrm{He}$ and $\mathrm{CO}_{2}$ drift gases.

\section{Computational Studies}

The analysis of the computational paradigm focuses first upon the seed geometries and general performance of the two SAMD protocols, followed by a detailed discussion of each peptide isomer. The discrepancies between the relative energies of varying conformations between DFT and CHARMM are highlighted, as well as deviations in the SAMD structures and the local minima obtained by DFT. Finally, the performance of the PA, EHSS and TM approximations for determining collision cross sections are compared along with the sensitivity of the methods and the ability to capture essential trends within the experimental data.

\section{OK DFT Optimized Structures and Protonation State Favorability}

For each of the tripeptides, varying protonation states may exist that impact the geometry (Figure 1): N-terminus (designated as A-state), tryptophan side chain (B-state),

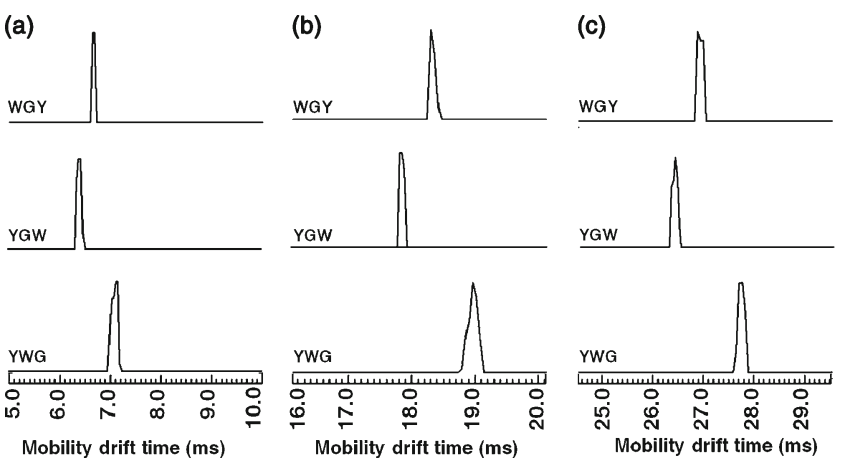

Figure 2. IMS spectra of YWG, WGY, and $Y G W(m / z 425)$ in (a) $\mathrm{He}$, (b) $\mathrm{N}_{2}$, and (c) $\mathrm{CO}_{2}$ drift gases. The horizontal axis displays the mobility drift time range 5-10 ms for the (a) He drift gas spectra; 16-20 ms for the (b) $\mathrm{N}_{2}$ drift gas spectra; and 25-30 ms for the (c) $\mathrm{CO}_{2}$ drift gas spectra. 
tyrosine side chain (C-state), as well as a number of zwitterionic states and those that result from protonation of the protein backbone $-\mathrm{NH}-$ and $-\mathrm{C}=\mathrm{O}$ groups. Initial DFT geometry optimizations were performed for both "elongated" and "compact" conformations of the tripeptides in the aforementioned protonation states. The zwitterionic forms are energetically unfavored in all isomers, with an absence of local minima in many cases. For each isomer, the (A) protonation state is the most favored by DFT (irrespective of density functional used). While normally this result would lead to the study of only the (A) state, it is important to query whether SAMD and subsequent $\Omega$ calculations will in-fact lead to the (A) state conformations having the best agreement with the experimental data. As such, the results for the (A) state, as well as the (B) and (C) protonation states, (which should not be contributing to the experimental data) are presented.

\section{General Observations Regarding SAMD}

Using the DFT optimized geometries for each protonation state as a starting point, SAMD was performed so as to allow each isomer to fully explore its conformational PES and determine a suitable set of conformations that are thermally populated at $473 \mathrm{~K}$. The resulting SAMD structures were independent of the initial conformation utilized (whether 'elongated' or 'compact'), indicating that both SAMD protocols, to a first approximation, thoroughly explored the conformational PES. In general, protocol \#1, which had the longest heating, equilibration, and cooling times resulted in a broader distribution of low-energy SAMD structures than protocol \#2. Moreover, while some of the structures resulting from protocol \#2 were very similar to those predicted by protocol $\# 1$, it was evident that many structures were missing from the conformational space. These observations are highlighted in Figure 3, which plots the relative energies of the 20 lowest energy conformations of the A-state of YGW (protonation at the $\mathrm{N}$ terminus) against the radius of gyration, $R_{\mathrm{gyr}}$, defined as the root-mean-square-deviation (RMSD) of the individual atoms from the center of mass within each conformation. The radius of gyration is a good measure of structural compactness and it is apparent in Figure 3 that at least five configurations are within $3 \mathrm{kcal} / \mathrm{mol}$ of the lowest energy

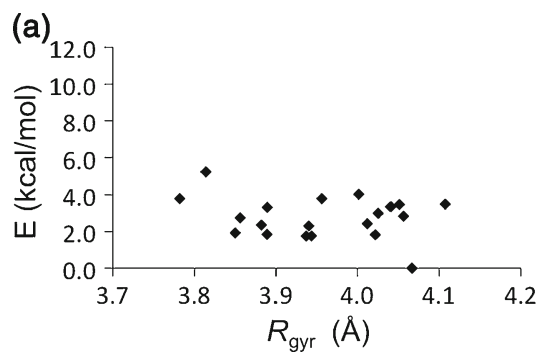

structure from protocol $\# 1$, while only one is obtained from protocol \#2. The same lowest-energy structure is obtained from both protocols; however, the distribution of conformations that lie within the thermally populated Boltzman distribution is very different. These data clearly illustrate a strong dependence of the conformational space upon the length of the equilibration, heating, and cooling times. Longer times for each of these operational parameters should therefore be adopted and protocol performance should be routinely examined so as to ensure a robust conformational sampling. Within this work, the SAMD simulations typically resulted in 1-4 structures that were likely candidates for thermal population.

To determine the structures that should be used in the calculation of $\Omega$, the SAMD data is typically used in one of three ways. First, structures within the Boltzmann distribution at the experimental temperature can be taken from the CHARMM energy distribution and used to determine $\Omega$. Second, DFT single point calculations can be performed on the SAMD structures so as to have a more accurate energy distribution that is used to select the thermally populated geometries. Finally, DFT geometry optimizations can be performed using the SAMD structures as starting points so as to find minima on the quantum mechanical conformational PESs, and collision cross section calculations can be performed on these geometries. For each isomer, we have performed the full suite of calculations, the results of which are discussed below.

\section{Determination of WGY Conformations at $473 \mathrm{~K}$}

Starting from the $0 \mathrm{~K}$ DFT optimized structure, Figure 4 presents the two low-energy structures determined by SAMD at $473 \mathrm{~K}$ for protonation state $\mathrm{A}$ of the WGY isomer. Each SAMD conformation exhibits H-bonding between the $\mathrm{N}$-terminus and the $\mathrm{O}$-atom within the tyrosine side chain. The structure WGY(A)-2a, also contains an interaction between the backbone O-atom of glycine and the $\mathrm{N}$-terminal $\mathrm{H}$-atom wherein the aromatic side-chains are nearly perpendicular to each other. The classical CHARMM energy difference between the two structures is $0.31 \mathrm{kcal} /$ mol [WGY(A)-2b lowest]. Single point B3LYP calculations with the 6-31G* and aug-cc-pVDZ basis sets, however, predict energy differences of $3.59 \mathrm{kcal} / \mathrm{mol}$ and $1.56 \mathrm{kcal} /$ mol, respectively, with WGY(A)-2a lowest in energy. The

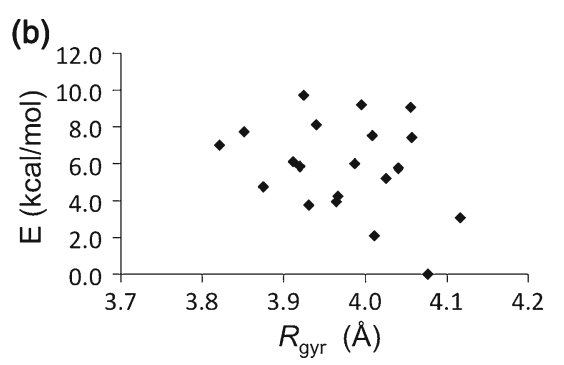

Figure 3. Relative energy of the 20 lowest energy conformations from protocol \#1 (a) and protocol \#2 (b) within the SAMD simulations for YGW(A), plotted against the radius of gyration, $R_{\mathrm{gyr}}$. 


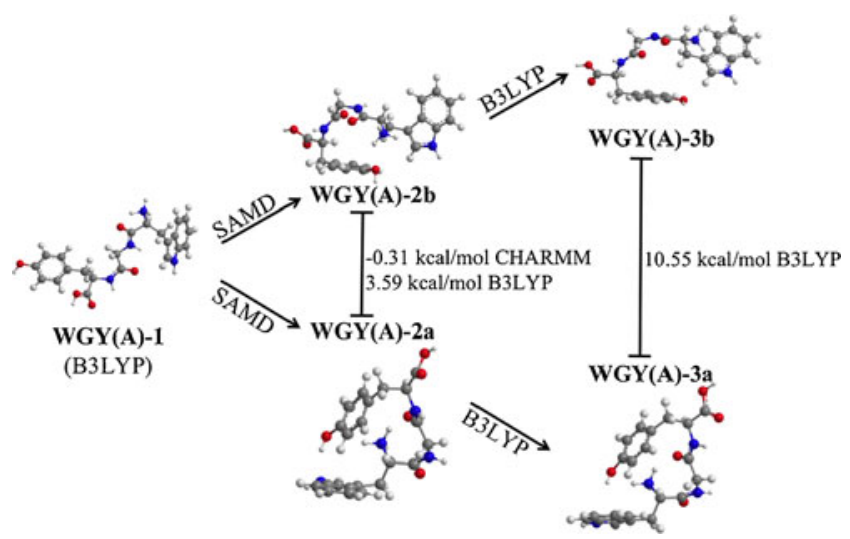

Figure 4. Initial B3LYP seed geometry of WGY in the A protonation state [WGY(A)-1], the resulting SAMD structures [WGY(A)-2a, WGY(A)-2b], and the B3LYP reoptimized geometries [WGY(A)-3a, WGY(A)-3b].

energy differences between conformations is an important issue, as in most cases, the average cross section of the lowest $2 \mathrm{kcal} / \mathrm{mol}$ structures in a given conformational family is used for comparison with experiment $[55,56]$. Moreover, the experimental peak widths for WGY in He and $\mathrm{CO}_{2}$ drift gases indicate non-interconverting conformations with nearly identical mobilities (and presumably similar energies), that can be 'teased apart' under varying drift gas conditions. Assuming that the drift gas does not effect the distribution of conformations that are present under the experimental conditions, then the basis set dependence of the single point energies becomes significant to the typical user if only single point calculations are going to be performed (vide infra). Subsequent DFT reoptimization of the SAMD structures with the $6-31 \mathrm{G}^{*}$ basis resulted in two new structures that are characterized by slight rotation of the tryptophan side chains that increases the energy separation to $10.55 \mathrm{kcal} / \mathrm{mol}$ (WGY(A)-3a lowest). Note that the lack of dispersion within the B3LYP functional does not appear to be relevant in this system, as the closed conformations predicted by SAMD are maintained upon geometry optimization.

Although the $\mathrm{B}$ and $\mathrm{C}$ protonation states are not expected to contribute to the experimental data, they are included within the computational study so as to determine whether the calculated collision cross sections can a priori indicate which protonation state contributes to the spectral data. Similar trends in the theoretical data are observed for both B and $\mathrm{C}$ states, wherein large deviations are observed in the energetic distributions of the conformations depending on the method of calculating the energy (CHARMM versus DFT). Further geometric relaxation occurs upon B3LYP reoptimization of the SAMD structures leading to new lower-energy minima. Within the B protonation state, SAMD identified a single, compact structure with H-bonding between the glycine backbone oxygen and $-\mathrm{NH}$ of the tryptophan side chain. B3LYP reoptimization of the SAMD geometry resulted in significant rotation of the tyrosine ring.
Similarly, SAMD identified one structure for protonation state $\mathrm{C}$, containing an $\mathrm{H}$-bond between the tryptophan backbone oxygen and the $\mathrm{H}$-atom bound to oxygen on the tyrosine side chain. Further information on specific structural parameters is available within the Online Resource.

Based upon the data above, it is evident that B3LYP (used with either the $6-31 \mathrm{G}^{*}$ or aug-cc-pVDZ basis sets) clearly disagrees with the energy ordering of the WGY conformations compared with that calculated by the CHARMM force field. To further complicate matters, it is well-established that DFT energies are highly sensitive to the particular combination of exchange and correlation energy functionals, and it is unclear if LDA, GGA, meta-GGA, and hybrid functionals will behave similarly, or if certain functionals will have better agreement with the classical energy distributions. Toward this end, the five lowest energy conformations from protocol \#1 of WGY(A) were subjected to single point energy calculations using PBE, BLYP, TPSS, VSXC, B3LYP, PBE1PBE, and TPSSH density functionals using the $6-31 \mathrm{G}^{*}$ basis set. The classic energy distributions are consistently much smaller than those calculated by DFT, and there is no agreement between CHARMM and any of the DFT functionals regarding which conformation is the lowest in energy. While some deviations in the energy distributions are observed between density functionals, the various DFT implementations typically agree on the lowest and highest energy conformations.

Figure 5 illustrates that for the five conformations of WGY(A) $(2 \mathrm{a}-2 \mathrm{e})$, all density functionals agree that $2 \mathrm{a}$ is the lowest energy conformation, and all but the VSXC functional agree that $2 \mathrm{e}$ is the highest energy. In contrast, CHARMM predicts $2 \mathrm{~b}$ to have the lowest in energy and $2 \mathrm{~d}$ to have the highest in energy. The general agreement of the varying density functionals with respect to the lowest energy structures within the spread of WGY conformations is promising for applications within IMS. However, the difference between the DFT and CHARMM energetic distributions remains disturbing, and is highlighted by structural deviations between the SAMD structures and those reoptimized by DFT.

\section{Determination of YGW Conformations at $473 \mathrm{~K}$}

SAMD predicts four low-energy structures for protonation state A within the Boltzmann distribution. All four structures are compact and exhibit H-bonding between the N-terminal hydrogen and the double-bonded oxygen on the C-terminus (Figure 6). Two of the four structures, $\mathrm{YGW}(\mathrm{A})-2 \mathrm{a}$ and YGW(A)-2b, exhibit H-bonding between the N-terminal Hatom and the first backbone oxygen. At the same time, YGW(A)-2c and YGW(A)-2 d show H-bonding between the hydrogen bound to the backbone nitrogen on tryptophan and the backbone oxygen on tyrosine. CHARMM predicts that all conformations exist within $2 \mathrm{kcal} / \mathrm{mol}$ of each other. Single point B3LYP/6-31G* calculations, instead, predict an $8 \mathrm{kcal} / \mathrm{mol}$ spread in the energies, with $\mathrm{YGW}(\mathrm{A})-2 \mathrm{c}$ 


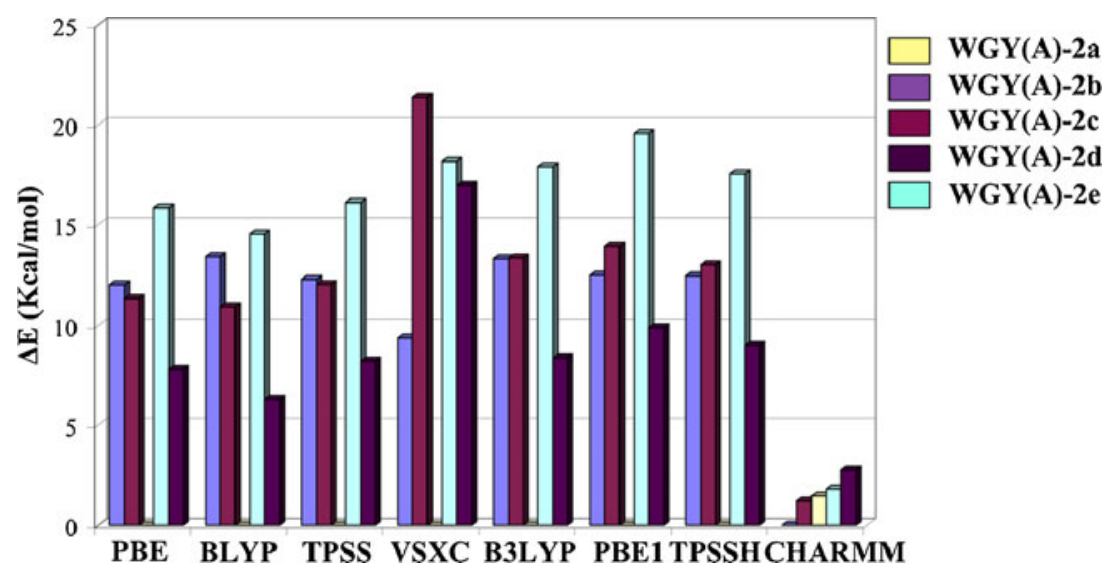

Figure 5. Relative energy differences (kcal/mol) for WGY(A)-2a through WGY(A)-2e, assessed by GGA, PBE, BLYP, TPSS, VSXC, B3LYP, PBE1PBE (labeled PBE1), and TPSSH density functionals in addition to the classical energy determined by the CHARMM force field.

the lowest, followed by $\mathrm{YGW}(\mathrm{A})-2 \mathrm{~b}(+0.86 \mathrm{kcal} / \mathrm{mol})$, YGW(A)-2a (+2.91 kcal/mol), YGW(A)-2d (+8.00 kcal/ mol). Similar results are obtained using the aug-cc-pVDZ basis that do not impact the averaging of conformational cross section values relative to the $6-31 \mathrm{G}^{*}$ basis (see Online Resource). DFT reoptimization led to slight reorientation of the tryptophan and tyrosine side chains, but the four separate local minima were maintained. Of the reoptimized structures, YGW(A)-3a had the lowest energy, followed by YGW(A)-3c $(+2.19 \mathrm{kcal} / \mathrm{mol}), \mathrm{YGW}(\mathrm{A})-3 \mathrm{~d}(+3.29 \mathrm{kcal} / \mathrm{mol})$, and YGW(A)-3b (+3.89 kcal/mol).

Protonation at tryptophan (to form state B) resulted in two SAMD conformations that are characterized by H-bonding between the tyrosine side chain oxygen and $\mathrm{H}$-atom bound to the tryptophan side chain nitrogen, as well as H-bonding between the tyrosine backbone oxygen and the hydrogen
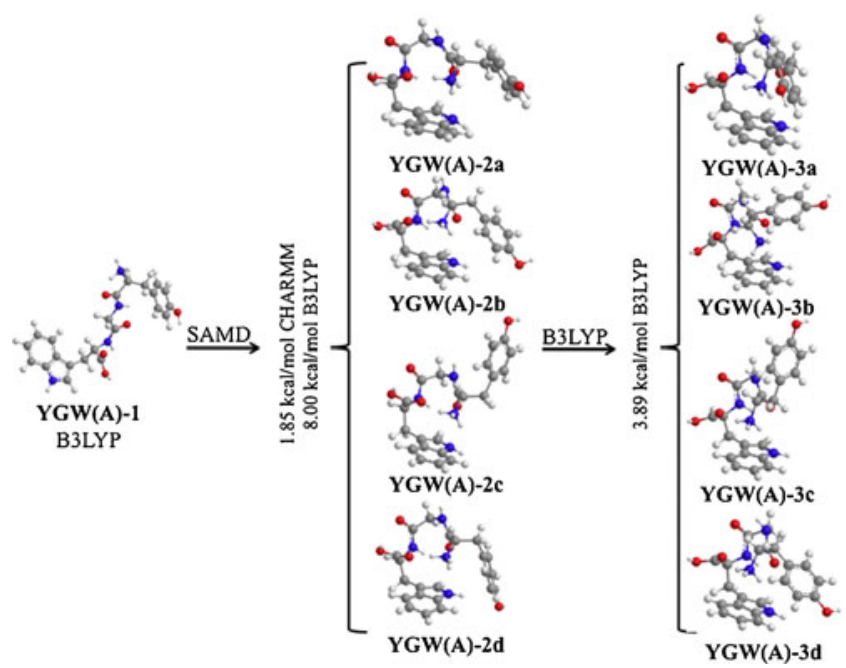

Figure 6. Initial B3LYP seed geometry of the A protonation state of $Y G W$ [YGW(A)-1], the resulting SAMD low energy structures $[Y G W(A)-2 a \ldots 2 d]$, and the B3LYP reoptimized geometries [YGW(A)-3a...3 d]. bound to the tryptophan backbone nitrogen (see Online Resource). B3LYP reoptimization of the SAMD structures led to pronounced differences in the position of tryptophan side chain. A single structure for state $\mathrm{C}$ was predicted, which exhibits strong $\mathrm{H}$-bonding between the $\mathrm{H}$-atom bound to the side chain oxygen of tyrosine and the C-terminal double bonded oxygen, and between the $\mathrm{C}$-terminal hydrogen and glycine backbone oxygen. DFT reoptimization did not significantly change the structure (see Online Resource).

\section{Determination of $Y W G$ Conformations at $473 \mathrm{~K}$}

Three SAMD structures were found to lie within the Boltzman distribution in the A protonation state. All structures contained various degrees of $\mathrm{H}$-bonding between the double-bonded C-terminal oxygen and N-terminal hydrogen, and the tryptophan back bone oxygen and the other N-terminal hydrogen (Figure 7). CHARMM predicts

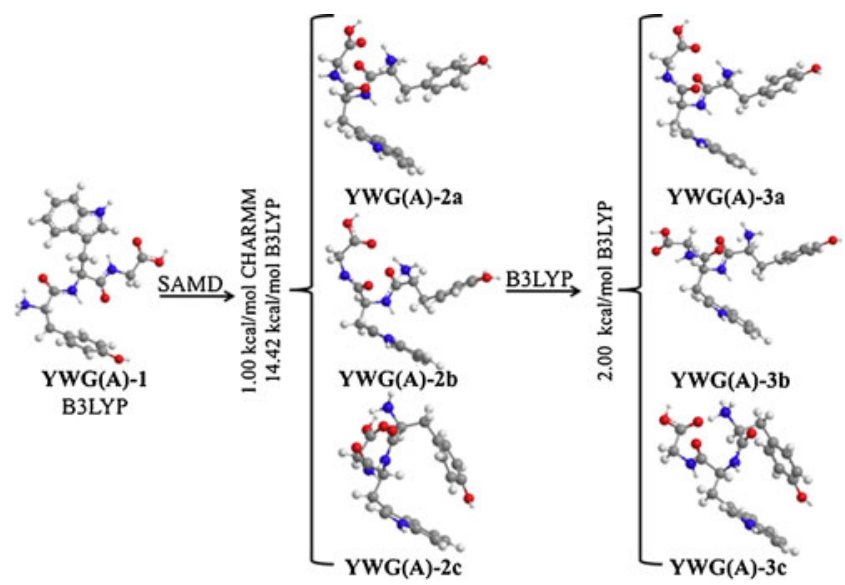

Figure 7. Initial B3LYP seed geometry of the A protonation state of YWG [YWG(A)-1], the low energy SAMD conformations $[Y W G(A)-2 a . .2 c]$, and $B 3 L Y P$ reoptimized structures [YWG(A)-3a...3c]. 
$2 \mathrm{c}$ to be the lowest energy, with the other two conformations lying within $1 \mathrm{kcal} / \mathrm{mol}$ in energy. Single point B3LYP/6$31 \mathrm{G}^{*}$ calculations also identified the YWG(A)-2c structure as the lowest in energy with $2 \mathrm{a} 8.29 \mathrm{kcal} / \mathrm{mol}$ and $2 \mathrm{~b}$ $14.42 \mathrm{kcal} / \mathrm{mol}$ higher in energy, respectively. Aug-ccpVDZ single point calculations decreased the energy differences slightly, but not enough to alter the distribution used in the calculation of $\Omega$. B3LYP reoptimization of the SAMD conformations had little effect upon YWG(A)-2a and YWG (A)-2c; however, the $\mathrm{C}$-terminus and tryptophan side chain reoriented significantly in YWG(A)-2b. The B3LYP YWG (A)-3c conformation is the lowest energy, with YWG(A)-3b and $\mathrm{YWG}(\mathrm{A})-3 \mathrm{c}$ lying within $2 \mathrm{kcal} / \mathrm{mol}$.

Two low-energy SAMD structures were identified when tryptophan is protonated (state B). Both structures have nearly parallel alignment of the tyrosine and tryptophan side chains and exhibit weak $\mathrm{H}$-bonding between the $-\mathrm{NH}$ on the tryptophan side chain and the side chain O-atom of tyrosine. DFT reoptimization causes pronounced changes in the Cterminus, tyrosine, and tryptophan side chain positions. In the case of the C-protonation state, SAMD found two nearly identical structures that exhibit strong H-bonding between the double bonded $\mathrm{C}$-terminal oxygen and the $-\mathrm{OH}$ of the tyrosine side chain. In addition, both structures have a $\mathrm{H}$ bond between the tryptophan backbone oxygen and the second hydrogen bound to the tyrosine side chain oxygen (see Online Resource). Reoptimization of the geometry using B3LYP yields a single structure, indicating that the CHARMM species were high-energy configurations linked to the same local minimum on the quantum mechanical PES.

\section{Comparison of Theoretical Versus Experimental Collision Cross Sections}

Typical computational strategies for correlating structure with cross section utilize diagnostic graphs of energy versus collision cross for candidate structures. Yet, as observed above, the energetic ranking is highly sensitive to the means by which the energy is calculated and, consequently, differences emerge between structures obtained from SAMD and those reoptimized using DFT. To investigate this further, the sensitivity of $\Omega$ was first examined using the SAMD structures but with the CHARMM versus DFT energy distributions. Let us define the percent deviation as the cross sections calculated using the B3LYP single point energy distribution $\left(\Omega_{\mathrm{B} 3 \mathrm{LYP}}\right)$ divided by the cross sections calculated using the CHARMM energy distribution $\left(\Omega_{\text {CHARMM }}\right)$ minus 1 , using each of the PA, EHSS or TM approximate methods. As observed in Figure 8, the percent deviation is generally negative by $2 \%-5 \%$, which indicates that DFT has an energetic preference for conformations that have slightly smaller cross sections relative to CHARMM. These data are essentially independent of basis set for the series of isomers studied here, as only one DFT energy distribution resulted in different "low energy" structures calculated using 6-31G* versus aug-cc-pVDZ. Given that

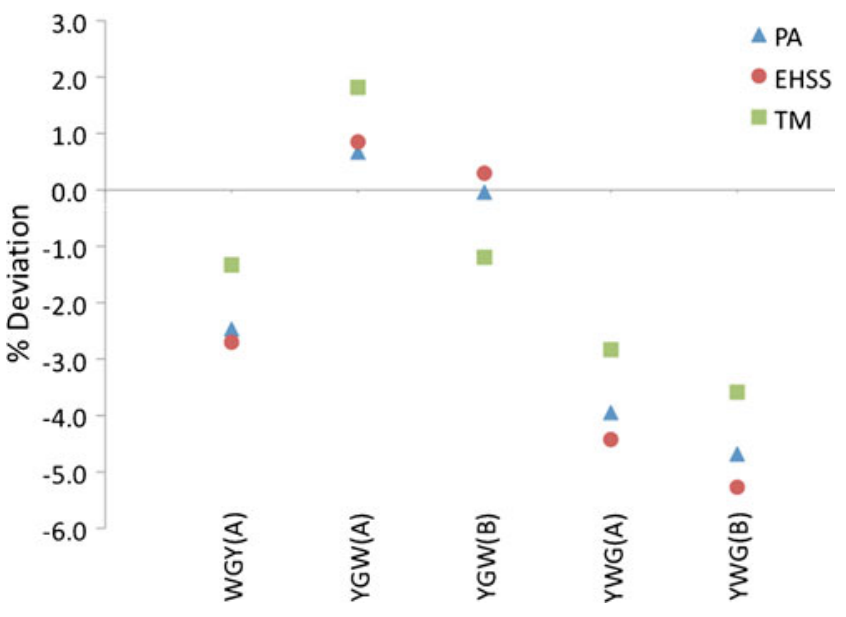

Figure 8. Percent deviation in calculated collision cross sections using the lowest energy SAMD structures calculated according to CHARMM and B3LYP using PA, EHSS, and TM (in $\mathrm{He}$ ) approximations. Percent deviation defined as $\Omega_{\mathrm{B} 3 \mathrm{LY}} /$ $\Omega_{\text {CHARMM }}-1$, where $\Omega_{\text {B } 3 L Y P}$ is the cross section calculated using the B3LYP energy distribution and $\Omega_{\text {CHARMM }}$ is calculated from the CHARMM energy distribution.

$2 \%$ accuracy within collision cross sections is typically desired within the literature, the deviation in the energy distributions calculated by CHARMM and DFT are unacceptably large. Interestingly, the TM approximation is typically the least sensitive to the deviations in energetic order within the same conformational family, though this may not be broadly observed among other systems.

Using visual inspection, DFT reoptimization of the SAMD structures often does not appreciably change the geometry, with the major structural changes being rotations of ring moieties. However, as seen in Figure 9 (which plots the percent deviation in collision cross section that occurs upon reoptimization) such alterations increase the collision cross section by $1 \%-4 \%$. In this case, the trajectory method is clearly much more sensitive than either PA or EHSS, which may indicate that reoptimization influences the TM results by exposing atoms within the tripeptide (e.g., $\mathrm{O}$ or N) that have a larger interaction with the drift gas (He used in Figures 8 and 9).

Figures 8 and 9 are useful for understanding the overall dependence of the calculated collision cross section upon energy distributions and subtle changes in analyte geometry; however, the typical MOBCAL user desires, if not quantitative accuracy in calculated $\Omega$, then a high degree of correlation between trends in experimental and theoretical $\Omega$. The experimental collision cross section data presented in Table 3 illustrates the clear separability of YWG from its isomeric counterparts. Depending upon the drift gas employed, WGY and YGW can also be separated from one another. Helium is typically the drift gas of choice and given its small polarizability $\left(0.204956 \times 10^{-30} \mathrm{~m}^{3}\right)$, the resulting collision cross sections are often compared with data obtained from either PA or EHSS as these methods 


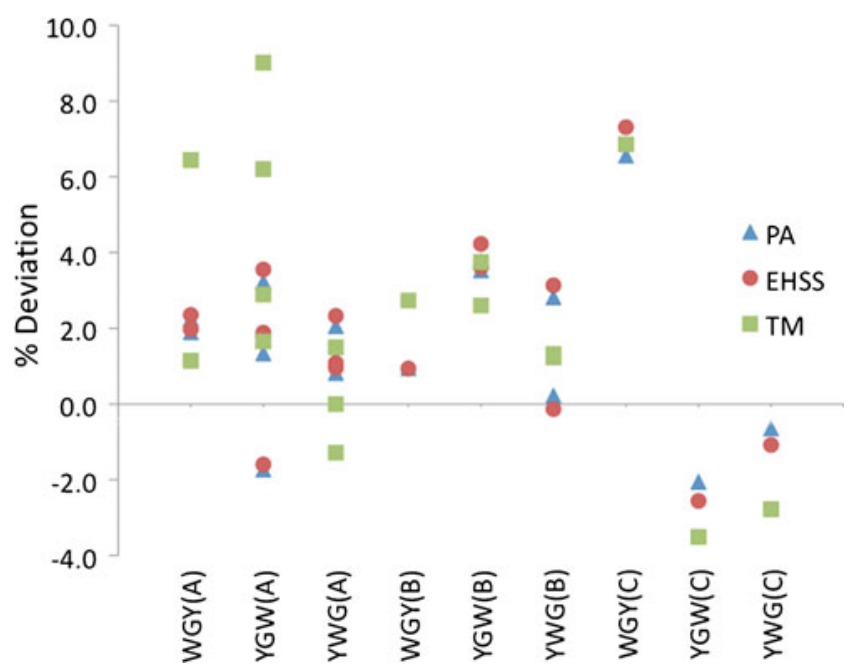

Figure 9. Percent deviation in calculated collision cross sections using the SAMD structures (with the B3LYP energetic distribution) and the B3LYP reoptimized structures using PA, EHSS, and TM (in $\mathrm{He}$ ) approximations. Percent deviation defined as $\Omega_{\mathrm{B} 3 L Y P} / \Omega_{\mathrm{SAMD}}-1$, where $\Omega_{\mathrm{B} 3 L Y P}$ is the cross section calculated using the B3LYP reoptimized structures and $\Omega_{\text {SAMD }}$ is calculated from the SAMD geometries using the B3LYP single point energy distributions.

ignore potential energy interactions between the drift gas and analyte. It is also useful to consider the zero-polarizability extrapolated $\Omega$ values. This extrapolation effectively removes the polarization and mass dependence of the drift gas upon the cross section, and as such represents the $\Omega_{\exp }$ value of each peptide as it exists in the gas phase, which is the condition under which the DFT and SAMD structures are determined. While the zero polarizability collision cross sections are $\sim 15 \AA^{2}$ smaller than those values obtained when He is used as a drift gas, in both cases the experimental cross sections are essentially the same for WGY and YGW isomers, while an $\sim 5 \%$ increase in cross section is observed for YWG.
Using the Projection Approximation, the calculated collision cross sections were compared using the CHARMM energy distribution, the B3LYP energy distribution that results from single point energy calculations of the SAMD conformations, and the B3LYP energy distributions from the DFT reoptimized structures (Figure 10). In comparison to the experimental data obtained in He drift gas (or the zero polarizability limit) only the cross sections calculated for protonation state A using the DFT reoptimized structures have the same trends in $\Omega$ as compared with experiment. Similar results are obtained when using the EHSS method, however, the magnitude of the calculated cross sections is $\sim 10 \%$ larger than when calculated with the Projection Approximation. Thus, the cross sections calculated with PA are closer to the zero polarizability extrapolated $\Omega$, while the $\Omega_{\text {EHSS }}$ are closer in magnitude to the experimental cross sections obtained using $\mathrm{He}$ as a drift gas (Table 4). In particular, when the DFT reoptimized structures are used, $\Omega_{\text {EHSS }}$ for WGY is 139.6, while for YGW it is 140.4 and for YWG the calculated value is $145.9 \AA^{2}$. Though closest to the experimental data, these cross section values are $2-4 \%$ too large relative to those obtained experimentally using $\mathrm{He}$ as a drift gas.

These data clearly illustrate that DFT reoptimization of the SAMD structures is required in order to have qualitatively correct trends in the collision cross sections within these moderately flexible analytes. Moreover, they are also consistent with the initial observation that the (A) protonation state is energetically preferred within the initial DFT calculations of the various protonation sites of each tripeptide. In the case of $\mathrm{YWG}$, reoptimization provided three different conformations for the (A) protonation state that are within $2 \mathrm{kcal} / \mathrm{mol}$ of one another, which agrees well with the overly broad peak widths in the IMS spectra for YWG in $\mathrm{He}$ and $\mathrm{CO}_{2}$ drift gases. Though the IMS spectra of WGY in $\mathrm{He}$ and $\mathrm{CO}_{2}$, as well as $\mathrm{YGW}$ in $\mathrm{N}_{2}$ drift gases also indicate non-interconverting conformations with nearly identical $\Omega$, DFT does not result in a sufficient number of low-energy

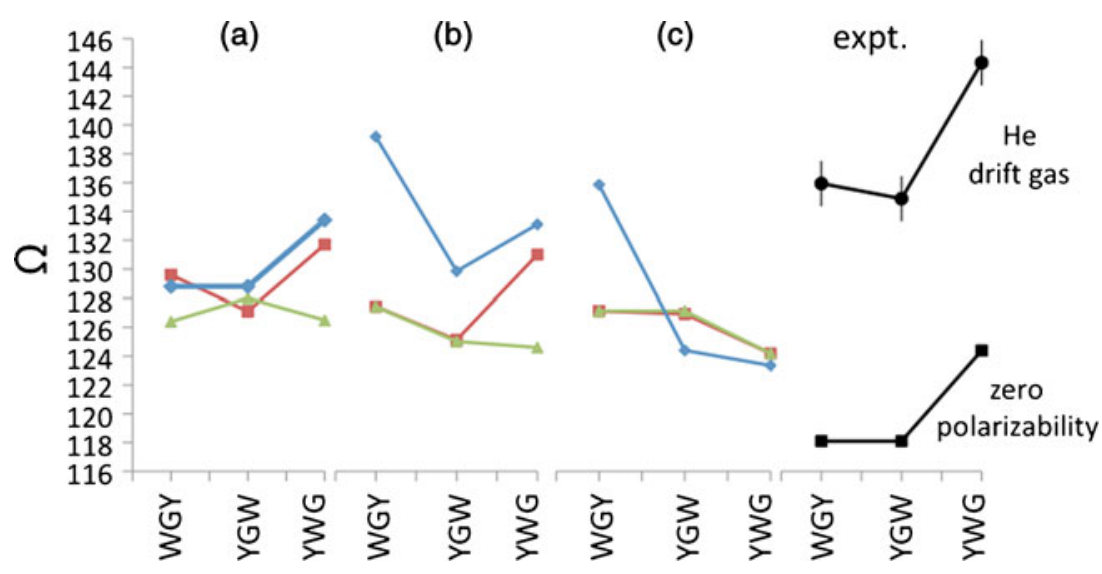

Figure 10. Collision cross sections obtained using the Projection Approximation for protonations states (a)-(c) using the CHARMM energy distribution (green), the B3LYP energy distribution using the SAMD structures (red), and the B3LYP reoptimized structures (blue). The data that follows the same experimental trend in bold. 
Table 4. Calculated Collision Cross Sections from the B3LYP Reoptimized SAMD Geometries, $\Omega_{\mathrm{PA}}, \Omega_{\mathrm{EHSS}}$, and $\Omega_{\mathrm{TM}}$ (in $\AA^{2}$ ), using eqs $4-6$, Respectively, in Comparison to the Experimental Values $\Omega_{\exp }$ for the A Protonation States of WGY, YGW, and YWG

\begin{tabular}{llll}
\hline & WGY(A)-3a & YGW(A)-3a & $<$ YWG(A)-3 $\rangle_{\text {avg }}$ \\
\hline$\Omega_{\text {PA }}$ & 128.8 & 128.8 & 133.4 \\
$\Omega_{\text {EHSS }}$ & 139.6 & 140.4 & 145.9 \\
$\Omega_{\exp }$ zero polarizability & 118 & 118 & 124 \\
$\Omega_{\mathrm{TM}}(\mathrm{He})$ & 123.8 & $124.4 /$ & 130.7 \\
$\Omega_{\exp }(\mathrm{He})$ & $135.0 \pm 1.5$ & $134.0 \pm 1.6$ & $143.0 \pm 1.1$ \\
$\Omega_{\mathrm{TM}}\left(\mathrm{N}_{2}\right)$ & 134.6 & 134.8 & 141.6 \\
$\Omega_{\exp }\left(\mathrm{N}_{2}\right)$ & $170.0 \pm 0.2$ & $167.0 \pm 0.4$ & $177.0 \pm 0.4$ \\
$\Omega_{\mathrm{TM}}\left(\mathrm{CO}_{2}\right)$ & 146.2 & 143.9 & 154.5 \\
$\Omega_{\exp }\left(\mathrm{CO}_{2}\right)$ & $205.0 \pm 1.5$ & $200.0 \pm 3.1$ & $213.0 \pm 0.6$ \\
\hline
\end{tabular}

structures to reproduce the experimental observation. It is likely that a larger number of SAMD structures must be reoptimized using DFT so that a more complete ensemble of local minima is obtained for these isomers. However, this has not been pursued, given that separate $\Omega_{\exp }$ were not obtained from IMS for these non-interconverting forms.

In the final step of the computational analysis, we examine whether significant improvement in the calculated $\Omega$ values are obtained if a typical user utilizes the Trajectory Method to determine the collision cross sections. In principle, since the Trajectory Method imparts more physics into the description of the collision events between drift gas and the analyte, the accuracy of the calculated collision cross sections should be significantly improved relative to simpler methods like PA and EHSS. As in the PA and EHSS results, only the (A) protonation state results in trends within $\Omega_{\mathrm{TM}}$ that mimic the experimental separability of the three peptide isomers. However, as observed in Table 4, the numerical accuracy of the calculated cross sections is not improved when "off-the-shelf" Lennard-Jones potentials are used to describe the interaction between drift gas and analyte. In the absence of fitting the LJ potentials to reproduce the experimental data, $\Omega_{\mathrm{TM}}$ calculated using the default $\mathrm{He}$ parameters in MOBCAL results in values that are $4 \%$ larger than the experimental zero polarizability extrapolated collision cross sections, and $\sim 8 \%$ smaller than the experimental cross sections obtained in $\mathrm{He}$ drift gas. Given that experimental drift gas polarizabilities were used within the MOBCAL calculation, the correct overall increase in cross sections is observed as the drift gas changes from $\mathrm{He}$, to $\mathrm{N}_{2}$, to $\mathrm{CO}_{2}$ within the TM data. However, the relative separability of the isomers changes as a function of drift gas within the IMS experiment, a feature that is not reflected within the TM data. This indicates that this feature of the experimental data is more sensitive to the interaction potentials that describe the collisions between the drift gas and various atoms within the analyte. Fitting of the individual LJ terms for each drift gas and the atoms within an analyte is beyond the scope of this work, but these data point to an area of much needed improvement within the field in order for the TM to be routinely used to quantitatively determine collision cross sections for proposed analytes from molecular simulation.

\section{Conclusions}

The present work has critically examined the accepted computational paradigm for obtaining gas phase geometries of analytes at experimental conditions relevant to IMS experiments, as well as the various approximations for determining collision cross sections therein. The analytes studied include the tripeptides WGY, YGW, and YWG, which have modest conformational flexibility $(\sim 10$ freely rotating bonds). While benchmarking studies have previously focused on very rigid analytes and large biomolecules, the best way to obtain accurate high temperature structures for molecules with an intermediate degree of flexibility has not been thoroughly investigated, nor has the performance of the PA, EHSS, and TM approximations for calculating their collision cross sections.

The SAMD protocol has been shown to be a good tool for exploring the conformational PES of these analytes and is an appropriate mechanism for providing initial guesses for DFT geometry optimization. However, the resulting conformational space is highly dependent upon the specific simulation protocol employed and it is recommended that it be benchmarked so as to ensure a robust set of thermally populated conformations. Investigation of a wide variety of density functionals revealed that most DFT methods agree on the lowest energy and highest energy geometries within a given set of SAMD conformations. However, the energy distributions and ranking of the lowest energy conformations obtained from the CHARMM force field did not agree with DFT. Further, direct use of the 'low-energy' SAMD geometries in calculations of the collision cross section did not yield values that correlated with experimental trends in the tripeptide separability. DFT reoptimization of the SAMD geometries, to yield local minima on the conformational PES of the analyte and subsequent calculation of the collision cross section, resulted in cross section values that were in good agreement with the relative separability of the WGY, YGW, and YWG analytes. Moreover, these data are consistent with the initial DFT determination that the (A) protonation state is the most energetically favored and as such should be the only protonation state contributing to the experimental data. As such, we conclude that for the specific systems studied here, the CHARMM force field is not accurate enough to be used to determine gas phase geometries (and energy distributions) used within $\Omega$ calculations. The EHSS method resulted in values that were $2 \%-$ $4 \%$ larger than those obtained experimentally, using $\mathrm{He}$ as a drift gas, while the cross sections calculated using the Projection Approximation were the closest to the zero polarizability extrapolated experimental values. Use of the Trajectory Method did not improve agreement with experimental data, as the specific interaction potentials were not optimized for the system under study. The results of this work indicate that study of moderately flexible analytes present their own pitfalls relative to less flexible molecules and detailed comparisons of the performance of methods 
used to calculate gas phase geometries and the performance of the PA, EHSS and TM approximations are vitally needed within the literature.

\section{Acknowledgments}

The authors thank Dr. Gerhard Munske for the peptide synthesis and Dr. Bill Siems for his help with experimental data analysis. N.L.Z. was supported in part by a research grant from the U.S. NIH 8RRR020046B R33RR020046 and grant $11 \mathrm{H} 24520762$ from Washington State University. C.L.C. was supported in part by grant 0731306 from the U.S. National Science Foundation. B.C.H. was supported by the NSF Research Experiences for Undergraduates (REU) program grant 0851502 . A.E.C. was supported in part with start-up funds from Washington State University.

\section{Online Resource}

Cartesian coordinates of all structures along with their DFT energies and further collision cross section information may be found as an online resource. http://www. springer.com/chemistry/analytical+chemistry/journal/13361? changeHeader

\section{References}

1. Karasek, F. W.; Kim, S. H.; Rokushika, S. Plasma Chromatography of Alkyl Amines. Anal. Chem. 197, 50, 2013-2016 (1978).

2. Clemmer, D.E., Hudgins, R.R., Jarrold, M.F.: Naked Protein Conformations: Cytochrome $c$ in the Gas Phase. J. Am. Chem. Soc. 117, 10141-10142 (1995)

3. Dwivedi, P., Wu, C., Matz, L.M., Clowers, B., Siems, W.F., Hill Jr., H. H.: Gas-Phase Chiral Separations by Ion Mobility Spectrometry. Anal. Chem. 78, 8200-8206 (2006)

4. Hudgins, R.R., Mao, Y., Ratner, M.A., Jarrold, M.F.: Conformations of $\mathrm{Gly}_{\mathrm{n}} \mathrm{H}^{+}$and $\mathrm{Ala}_{\mathrm{n}} \mathrm{H}^{+}$peptides in the Gas Phase. Biophys. J. 76, 15911597 (1999)

5. Shumate, C.B., Hill Jr., H.H.: Coronaspray Nebulization and Ionization of Liquid Samples for Ion Mobility Spectrometry. Anal. Chem. 61, 601-606 (1989)

6. McLean, J.A., Ruotolo, B.T., Gillig, K.J., Russell, D.H.: Ion MobilityMass Spectrometry: A New Paradigm for Proteomics. Int. J. Mass Spectrom. 240, 301-315 (2005)

7. Shvartsburg, A.A., Li, F., Tang, K., Smith, R.D.: Characterizing the Structures and Folding of Free Proteins Using 2-D Gas-Phase Separations: Observation of Multiple Unfolded Conformers. Anal. Chem. 78, 3304-3315 (2006)

8. Dwivedi, P., Wu, P., Klopsch, S.J., Puxon, G.J., Xun, L., Hill Jr., H.H.: Metabolic Profiling by Ion Mobility Mass Spectrometry (IMMS). Metabolomics 4, 63-80 (2008)

9. Dwivedi, P., Bendiak, B., Clowers, B.H., Hill Jr., H.H.: Rapid Resolution of Carbohydrate Isomers by Electrospray Ionization Ambient Pressure Ion Mobility Spectrometry-Time-of-Flight Mass Spectrometry (ESI-APIMS-TOFMS). J. Am. Soc. Mass Spectrom. 18, 1163-1175 (2007)

10. Fernandez-Lima, F.A., Becker, C., Gillig, K., Russell, W.K., Nascimento, M.A.C., Russell, D.H.: Experimental and Theoretical Studies of $(\mathrm{CsI})_{\mathrm{n}} \mathrm{Cs}+$ Cluster Ions Produced by $355 \mathrm{~nm}$ Laser Desorption Ionization. J. Phys. Chem. A 112, 11061-11066 (2008)

11. von Helden, G., Wyttenbach, G.T., Bowers, M.T.: Conformations of Macromolecules i the Gas Phase: Use of Matrix-Assisted Laser Desorption Methods in Ion Chromatography. Science 267, 1483-1485 (1995)

12. Myung, S., Lorton, K.P., Merenbloom, S.I., Fioroni, M., Koeniger, S. L., Julian, R.R., Baik, M.-H., Clemmer, D.E.: Chirally Directed Formation of Nanometer-Scale Proline Clusters. J. Am. Chem. Soc. 128, 10833-10839 (2006)
13. Myung, S., Lorton, K.P., Merenbloom, S.I., Fioroni, M., Koeniger, S. L., Julian, R.R., Baik, M.-H., Clemmer, D.E.: Evidence for Spontaneous Resolution of Icosahedral Proline. J. Am. Chem. Soc. 128, 1598815989 (2006)

14. Jarrold, M.F.: Peptides and Proteins in the Vapor Phase. Ann. Rev. Phys. Chem. 51, 179-207 (2000)

15. Valentine, S.J., Counterman, A.E., Clemmer, D.E.: A Database of 660 Peptide Ion Cross Sections: Use of Intrinsic Size Parameters for Bona Fide Predictions of Cross Sections. J. Am. Soc. Mass Spectrom. 10, 1188-1211 (1999)

16. Tao, L., McLean, J.R., McLean, J.A., Russell, D.H.: A Collision CrossSection Database of Singly-Charged Peptide Ions. J. Am. Soc. Mass Spectrom. 18, 1232-1238 (2007)

17. Hill Jr., H.H., Hill, C.H., Asbury, G.R., Wu, C., Matz, L.M., Ichiye, T.: Charge location on gas phase peptides. Int. J. Mass Spectrom. 219, $23-$ 27 (2002)

18. Williams, J.P., Bugarcic, T., Habtermariam, A., Giles, K., Campuzano, I., Rodger, P.M., Sadler, P.J.: Isomer Separations and Gas-Phase Configurations of Organoruthenium Anticancer Complexes: Ion Mobility Mass Spectrometry and Modeling. J. Am. Soc. Mass Spectrom. 20, 1119-1122 (2009)

19. Fernandez-Lima, F.A., Wei, H., Gao, Y.Q., Russell, D.H.: On the Structure Elucidation Using Ion Mobility Spectrometry and Molecular Dynamics. J. Phys. Chem. A 113, 8221-8234 (2009)

20. Tao, L., Dahl, D.B., Perez, L.M., Russell, D.H.: The Contributions of Molecular Framework to IMS Collision Cross-Sections of Gas-Phase Peptide Ions. J. Am. Soc. Mass Spectrom. 20, 1593-1602 (2009)

21. Bleiholder, C., Osburn, S., Williams, T.D., Suahi, S., van Stipdonk, M., Harrison, A.G., Paizs, B.: Sequence-Scrambling Fragmentation Pathways of Protonated Peptides. J. Am. Chem. Soc. 130, 17774-17789 (2008)

22. Spangler, G.E.: Theory and Technique for Measuring Mobility Using Ion Mobility Spectrometry. Anal. Chem. 65, 3010-3014 (1993)

23. Revercomb, H.E., Mason, E.A.: Theory of plasma chromatography/ gaseous electrophoresis. Anal. Chem. 47, 970-983 (1975)

24. Spangler, G.E.: Expanded theory for the resolving power of a linear ion mobility spectrometer. Int. J. Mass Spectrom. 220, 399-418 (2002)

25. Kanu, A.B., Gribb, M.M., Hill Jr., H.H.: Predicting Optimal Resolving Power for Ambient Pressure Ion Mobility Spectrometry. Anal. Chem. 80, 6610-6619 (2008)

26. Siems, W.F., Wu, C., Tarver, E.E., Hill Jr., H.H., Larsen, P.R., McMinn, D.G.: Measuring the Resolving Power of Ion Mobility Spectrometers. Anal. Chem. 66, 4195-4201 (1994)

27. Asbury, R.G., Hill Jr., H.H.: Evaluation of ultrahigh resolution ion mobility spectrometry as an analytical separation device in chromatographic terms. J. Microcolumn Sep. 12, 172-178 (2000)

28. Asbury, R.G., Hill Jr., H.H.: Using Different Drift Gases to Change Separation Factors $(\longmapsto)$ in Ion Mobility Spectrometry. Anal. Chem. 72, 580-584 (2000)

29. Steiner, W.E., Klopsch, S.J., English, W.A., Clowers, B.H., Hill Jr., H. H.: Electrospray Ionization with Ambient Pressure Ion Mobility Separation and Mass Analysis by Orthogonal Time-of-Flight Mass Spectrometry. Rapid Commun. Mass Spectrom. 15, 2221-2226 (2001)

30. Steiner, W.E., Clowers, B.H., Hill Jr., H.H.: Rapid Separation of Phenylthiohydantoin Amino Acids: Ambient Pressure Ion-Mobility Mass Spectrometry (IMMS). Anal. Bioanal. Chem. 375, 99-102 (2003)

31. Steiner, W.E.C., Brian, H., Haigh, P.E., Hill Jr., H.H.: Secondary Ionization of Chemical Warfare Agent Simulants: Atmospheric Pressure Ion Mobility Time-of-Flight Mass Spectrometry. Anal. Chem. 75, 6068-6076 (2003)

32. Beegle, L.W., Kanik, I., Matz, L., Hill Jr., H.H.: Effects of Drift-Gas Polarizability on Glycine Peptides in Ion Mobility Spectrometry. Int. J. Mass Spectrom. 216, 257-268 (2002)

33. Becke, A.D.: Density-Functional Thermochemistry. III. The Role of Exact Exchange. J. Chem. Phys 98, 5648-5652 (1993)

34. Lee, C., Yang, W., Parr, R.G.: Development of the Colle-Salvetti Correlation-Energy Formula into a Functional of the Electron Density. Phys. Rev. B 37, 785-789 (1988)

35. Hehre, W.J., Ditchfield, R., Pople, J.A.: Self-Consistent Molecular Orbital Methods. XII. Further Extensions of Gaussian-Type Basis Sets for Use in Molecular Orbital Studies of Organic Molecules. J. Chem. Phys. 56, 2257-2261 (1972)

36. Frisch, M. J.; Trucks, H. B. S. G. W.; Scuseria, G. E.; Robb, M. A.; Cheeseman, J. R.; Montgomery, J. A. Jr.; Vreven, T.; Kudin, K. N.; Burant, J. C.; Millam, J. M.; Iyengar, S. S.; Tomasi, J.; Barone, V.; Mennucci, B.; Cossi, M.; Scalmani, G.; Rega, N.; Petersson, G. A.; 
Nakatsuji, H.; Hada, M.; Ehara, M.; Toyota, K.; Fukuda, R.; Hasegawa, J.; Ishida, M.; Nakajima, T.; Honda, Y.; Kitao, O.; Nakai, H.; Klene, M.; Li, X.; Knox, J. E.; Hratchian, H. P.; Cross, J. B.; Bakken, V.; Adamo, C.; Jaramillo, J.; Gomperts, R.; Stratmann, R. E.; Yazyev, O.; Austin, A. J.; Cammi, R.; Pomelli, C.; Ochterski, J. W.; Ayala, P. Y.; Morokuma, K.; Voth, G. A.; Salvador, P.; Dannenberg, J. J.; Zakrzewski, V. G.; Dapprich, S.; Daniels, A. D.; Strain, M. C.; Farkas, O.; Malick, D. K.; Rabuck, A. D.; Raghavachari, K.; Foresman, J. B.; Ortiz, J. V.; Cui, Q.; Baboul, A. G.; Clifford, S.; Cioslowski, J.; Stefanov, B. B.; Liu, G.; Liashenko, A.; Piskorz, P.; Komaromi, I.; Martin, R. L.; Fox, D. J.; Keith, T.; Al-Laham, M. A.; Peng, C. Y.; Nanayakkara, A.; Challacombe, M.; Gill, P. M. W.; Johnson, B.; Chen, W.; Wong, M. W.; Gonzalez, C.; Pople, J. A. Gaussian 03, Revision D.02., Gaussian, Inc.: Wallingford, CT. (2004)

37. Dunning, T.H.: Gaussian basis sets for use in correlated molecular calculations. I. The atoms boron through neon and hydrogen. J. Chem. Phys 90, 1007-1023 (1989)

38. Brooks, B. R.; Brooks C. L. III; Mackerell, A. D. Jr.; Nilsson, L.; Petrella, R. J.; B.; Roux, B.; Won, Y.; Archontis, G.; Bartels, C.; Boresch, S.; Caflisch, A.; Caves, L.; Cui, Q.; Dinner, A. R.; Feig, M.; Fischer, S.; Gao, J.; Hodoscek, M.; Im, W.; Kuczera, K.; Lazaridis, T.; Ma, J.; Ovchinnikov, V.; Paci, E.; Pastor, R. W.; Post, C. B.; Pu, J. Z.; Schaefer, M.; Tidor, B.; Venable, R. M.; Woodcock, H. L.; Wu, X.; Yang, W.; York, D. M.; Karplus, M. CHARMM 09 J. Comput. Chem. 1545-1614 (2009)

39. Gao, B., Wyttenbach, T., Bowers, M.T.: Hydration of Protonated Aromatic Amino Acids: Phenylalanine, Tryptophan, and Tyrosine. $J$. Am. Chem. Soc 131, 4695-4701 (2009)

40. Liu, D., Seuthe, A.B., Ehrler, O.T., Zhang, X., Wyttenbach, T., Hsu, J. F., Bowers, M.T.: Oxytocin-Receptor Binding: Why Divalent Metals Are Essential. J. Am. Chem. Soc 127, 2024-2025 (2005)

41. Brocker, E.R., Anderson, S.E., Northrop, B.H., Stang, P.J., Bowers, M. T.: Structures of Metallosupramolecular Coordination Assemblies Can Be Obtained by Ion Mobility Spectrometry-Mass Spectrometry. J. Am. Chem. Soc 132, 13486-13494 (2010)

42. Perdew, J.P., Burke, K., Ernzerhof, M.: Generalized Gradient Approximation Made Simple. Phys. Rev. Lett. 77, 3865-3868 (1996)

43. Tao, J., Perdew, J.P.: Climbing the Density Functional Ladder: Nonempirical Meta- Generalized Gradient Approximation Designed for Molecules and Solids. Phys. Rev. Lett. 91, 146401-146405 (2003)

44. Voorhis, T.V., Scuseria, G.E.: A novel form for the exchangecorrelation energy functional. J. Chem. Phys. 109, 400-410 (1998)

45. Perdew, J.P., Burke, K., Ernzerhof, M.: Generalized Gradient Approximation Made Simple. Phys. Rev. Lett. 77(18), 3865-3868 (1997)

46. Staroverov, V., Scuseria, G.E., Tao, J., Perdew, J.P.: Comparative Assessment of a New Nonempirical Density Functional: Molecules and Hydrogen-Bonded Complexes. J. Chem. Phys. 119, 12129-12137 (2003)
47. Bylaska, E. J.; de Jong, W. A.; Govind, N.; Kowalski, K.; Straatsma, T. P.; Valiev, M.; Wang, D.; Apra, E.; Windus, T. L.; Hammond, J.; Nichols, P.; Hirata, S.; Hackler, M. T.; Zhao, Y.; Fan, P.-D.; Harrison, R. J.; Dupuis, M.; Smith, D. M. A.; Nieplocha, J.; Tipparaju, V.; Krishnan, M.; Wu, Q.; Van Voorhis, T.; Auer, A. A.; Nooijen, M.; Crosby, L. D.; Brown, E.; Cisneros, G.; Fann, G. I.; Fruchtl, H.; Garza, J.; Hirao, K.; Kendall, R.; Nichols, J. A.; Tsemekhman, K.; Wolinski, K.; Anchell, J.; Bernholdt, D.; Borowski, P.; Clark, T.; Clerc, D.; Dachsel, H.; Deegan, M.; Dyall, K.; Elwood, D.; Glendening, E.; Gutowski, M.; Hess, A.; Jaffe, J.; Johnson, B.; Ju, J.; Kobayashi, R.; Kutteh, R.; Lin, Z.; Littlefield, R.; Long, X.; Meng, B.; Nakajima, T.; Niu, S.; Pollack, L.; Rosing, M.; Sandrone, G.; Stave, M.; Taylor, H.; Thomas, G.; van Lenthe, J.; Wong, A.; Zhang, Z. NWChem A Computational Chemistry Package for Parallel Computers, Version 5.1.1. , Pacific Northwest National Laboratory: Richland, WA. (2008)

48. Mesleh, M. F.; Hunter, J. M.; Shvartsburg, A. A.; Schatz, G. C.; Jarrold, M. F. Structural Information from Ion Mobility Measurements: Effects of the Long Range Potential. J. Phys. Chem. A, Erratum, 101, 1608216086 (1997)

49. Shvartsburg, A.A., Jarrold, M.F.: An Exact Hard Spheres Scattering Model for the Mobilities of Polyatomic Ions. Chem. Phys. Lett. 261, 86-91 (1996)

50. Mack, E.J.: Average Cross-Sectional Areas of Molecules by Gaseous Diffusion Methods. J. Am. Chem. Soc 47, 2468-2482 (1925)

51. Jarrold, M.F., Constant, V.A.: Silicon cluster ions: Evidence for a structural transition. Phys. Rev. Lett. 67, 2994 (1992)

52. Clemmer, D.E., Hunter, J.M., Selimov, K.B., Jarrold, M.F.: Physical and Chemical Evidence for Metallofullerenes with Metal Atoms as Part of the Cage. Nature 372, 248-250 (1994)

53. Mesleh, M.F., Hunter, J.M., Shvartsburg, A.A., Schatz, G.C., Jarrold, M.F.: Structural Information from Ion Mobility Measurements: Effects of the Long Range Potential. J. Phys. Chem. 100, 1608216086 (1996)

54. Cuadros, F., Cachadina, I., Ahumada, W.: Determination of LennardJones Interaction Parameters Using a New Procedure. Mol. Eng. 6, 319 325 (1996)

55. Wyttenbach, T., Liu, D., Bowers, M.T.: Interactions of the Hormone Oxytocin with Divalent Metal Ions. J. Am. Chem. Soc. 130, 5993-6000 (2008)

56. Gidden, J., Bowers, M.T.: Gas-Phase Conformations of Deprotonated and Protonated Mononucleotides Determined by Ion Mobility and Theoretical Modeling. J. Phys. Chem. B 107, 12829-12837 (2003)

57. Steiner, W.E., English, W.A., Hill Jr., H.H.: Ion-Neutral Potential Models in Atmospheric Pressure Ion Mobility Time-of-Flight Mass Spectrometry IM(TOF)MS. J. Phys. Chem. A, 110, 1836-1844 (2006) 